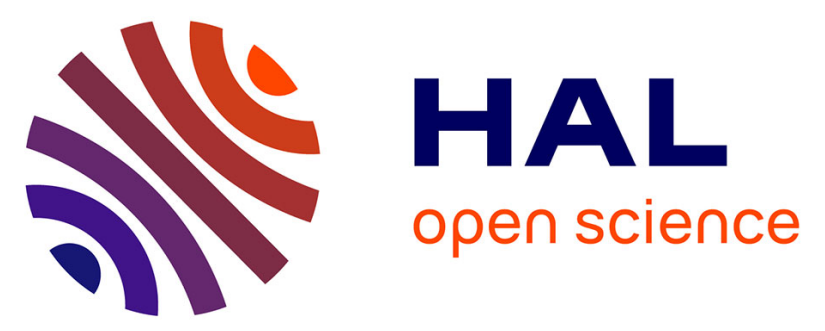

\title{
STRUCTURAL STUDY OF THE LAMELLAR TO COLUMNAR TRANSITION IN THERMOTROPIC LIQUID CRYSTALS : THE THERMOTROPIC CUBIC PHASE OF SOME PHASMIDIC MOLECULES
}

\author{
A. Levelut, Y. Fang
}

\section{To cite this version:}

A. Levelut, Y. Fang. STRUCTURAL STUDY OF THE LAMELLAR TO COLUMNAR TRANSITION IN THERMOTROPIC LIQUID CRYSTALS : THE THERMOTROPIC CUBIC PHASE OF SOME PHASMIDIC MOLECULES. Journal de Physique Colloques, 1990, 51 (C7), pp.C7-229-C7-236. 10.1051/jphyscol:1990722 . jpa-00231121

HAL Id: jpa-00231121 https://hal.science/jpa-00231121

Submitted on 1 Jan 1990

HAL is a multi-disciplinary open access archive for the deposit and dissemination of scientific research documents, whether they are published or not. The documents may come from teaching and research institutions in France or abroad, or from public or private research centers.
L'archive ouverte pluridisciplinaire HAL, est destinée au dépôt et à la diffusion de documents scientifiques de niveau recherche, publiés ou non, émanant des établissements d'enseignement et de recherche français ou étrangers, des laboratoires publics ou privés. 
COLLOQUE DE PHYSIQUE

Colloque C7, supplément au $n^{\circ} 23$, Tome 51, ler décembre 1990

STRUCTURAL STUDY OF THE LAMELLAR TO COLUMNAR TRANSITION IN THERMOTROPIC LIQUID CRYSTALS : THE THERMOTROPIC CUBIC PHASE OF SOME PHASMIDIC MOLECULES

\author{
A.M. LEVELUT and Y. FANG* \\ Laboratoire de physique des Solides, Bâtiment 510, Université \\ Paris-Sud, F-91405 Orsay Cedex, France \\ * Present address : Department of Biological Sciences, Brock University, \\ st Catherines, Canada
}

\title{
Résumé
}

Le polymorphisme thermotrope des molécules phasmidiques à quatre chaînes se compare à celui des systèmes lyotropes. En particulier, certaines séries d'homologues présentent une phase intermédiaire de symétrie $\operatorname{Im} 3 \mathrm{~m}$ entre une phase lamellaire et une phase colonnaire. Des phasmides à trois chaînes présentent cette même phase. Nous avons mesuré les paramètres de maille sur quelques composés purs ainsi que sur des mélanges binaires. Il est possible, à partir de ces données, de calculer les caractéristiques structurales telles que l'épaisseur d'une couche moléculaire et l'aire par chaîne pour un modèle d'organisation où deux labyrinthes feuilletés sont séparés par une surface minimale périodique.

\section{$\underline{\text { Abstract }}$}

The thermotropic mesomorphism of four-chains phasmids is similar to that of lyotropic systems. A cubic Im3m mesophase can be detected between a lamellar phase on one side and a columnar phase on the other side. Moreover, the same cubic phase is found for some three-chains phasmids. The lattice cell unit of this phase has been measured for several pure compounds and for binary mixtures. These measurements are consistent with a model of the molecular organisation in which two labyrinthes, each made of two molecular layers, are delimited by a periodic minimal balance surface. The layer thicknesses and the mean chain area are calculated on the basis of this model.

\section{I - INTRQDUCTION}

Almost all the liquid crystalline structures can be considered as periodically organised fluids.In lyotropic systems, the phase diagrams present areas of one-dimensionnal(1D) organization periodic stacks of infinite lamellae, and of two-dimensional (2D) networks (hexagonal assembly of infinite and parallel cylinders). Between these two different classes, corresponding to different topological arrays of the two media (hydrophobic and hydrophilic ones), an area of three-dimensional (3D) organized fluids can be found in the phase diagram $/ 1$. Within this last class, there are cubic lattices of different symmetries. Their lattice size $(\approx 10 \mathrm{~nm})^{3}$ is large in comparison with the molecular volume and the topology is " bicontinuous": a thin film of one medium makes the interface between two imbricated labyrinthes of the second medium. A theoretical approach of such bicontinuous cubic structures give a description of the interface between the two labyrinthes in term of minimal surfaces $/ 2 /$. Experimentally a structural analysis provides an image consistent with this approach at least for three different space groups /3/. Thermotropic liquid crystals present also lamellar and columnar structures. However, generally the shape of the molecule, disc or rod, drives the symmetry properties of the mesophases. nevertheless cubic mesophases have been put in evidence in some disc-like or rod-like molecules /4/ but the structural information is still very limited. A new class of molecules, the phasmids $15 /$, offer the opportunity of a more systematic study of topological transformations from the lamellae to the cylinders since such molecules present smectic $\mathrm{C}(\mathrm{SmC})$ and hexagonal columnar (Hex I) phases in the same series or even in the same derivative. The phasmidic molecules have an hybrid shape with a long rod-like core with one to three paraffinic chains grafted on each end. The transition between the lamellar and the cylindrical state is obtained with molecules of a core of five or six phenyl rings with four or three alkyloxy chains of about ten carbon atom length. This transition involves 
several steps corresponding either to $2 \mathrm{D}$ ribbon lattices or to different, hexagonal, rhombohedral or cubic 3D networks $/ 6 /$.Moreover, we have shown that the topology of the phase diagram is very similar in lyotropic and in phasmidic mesophases, provided that the chain to core volume ratio (for thermotropic systems) takes place of the solvent amount (in lyotropic ones) $\Pi /$. Therefore, a structural analysis of these mesophases can be driven based upon a comparison with the lyotropic mesophases of similar symmetry. In a first step we have selected the cubic mesophase since we have already identified the same symmetry in one three-chains compound and in five four-chains compounds. The space group is likely I $\mathrm{m} 3 \mathrm{~m}$ and the lattice parameter varies between 15 and $18 \mathrm{~nm}$.

In the next section, we present the different molecules and their polymorphism. Then we analyse the structural features of the cubic mesophase, we compare this phase with other mesophases of known structure and we also present some binary phase diagrams. At last, we discuss a model for microscopical organisation based upon a Schwarz $3 D$ periodic minimal balance surface of $P$ type.

\section{II- CUBIC MESOPHASES IN PHASMIDIC COMPOUNDS}

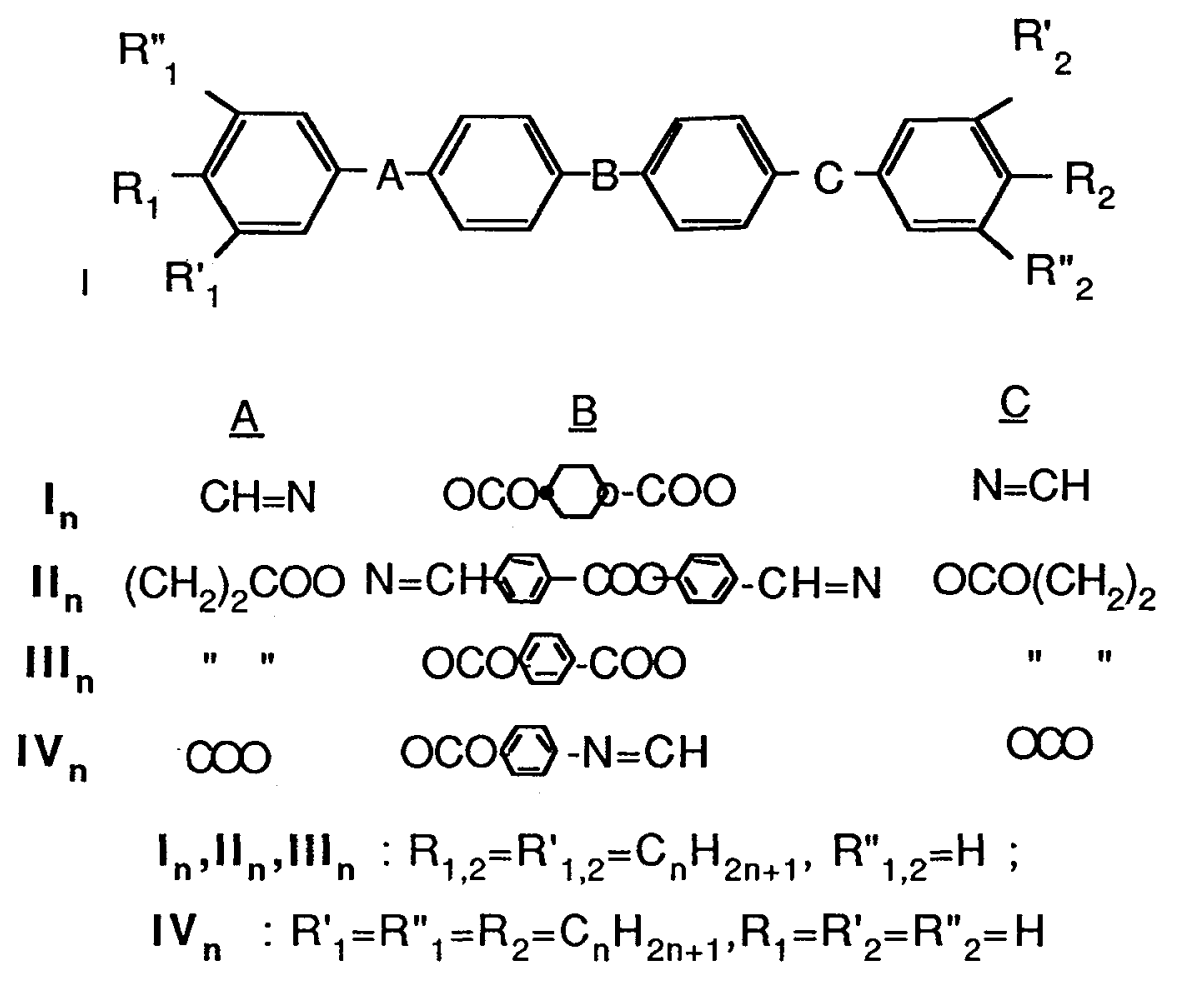

Fig. 1. General chemical structure of phasmidic molecules

The first phasmidic cubic mesophase was put in evidence in a biforked mesogen (four-chains) with a central cyclohexyl ring in the rod-like core Serie I /8/ (fig. 1). Four other biforked compounds present also a cubic mesophase, two belong to serie I, the two others correspond to different cores $\Pi / 9 /$ and III $/ 10 \%$. The polymorphism is nearly the same for these five compounds : on heating crystalline, $\mathrm{SmC}$, cubic and isotropic phases are seen; on cooling these compounds from the isotropic liquid a metastable hexagonal columnar mesophase appears first and transforms itself at lower temperature into the cubic phase. The smC phase is metastable in serie $\mathbf{I}$, and cannot be detected by X-ray diffraction. On the contrary, it is stable in compounds II $_{10}$ and III8 and it can appear also together with the high temperature monotropic hexagonal mesophase in compound III8 depending on the cooling rate. The metastable hexagonal mesophase(Hex II) has been identified by X-ray diffraction only on two 
compounds of serie I. This complex behaviour puts in evidence the slight differences of stability that could exist between these different phases. Another compound /11/ has been studied and presents a simpler mesomorphism with only a cubic phase stable over about $100 \mathrm{~K}$ of temperature range. This compound has a rod-like core constituted of five phenyl rings, two dodecyloxy chains are grafted in meta position on one end while a unique dodecyl chain is in para position on the other end (IV 12 ).

The SmC /12/, for short chains, and the Hex I phase /13/, for long chains, have been extensively studied in several series, in addition the mesomorphism of other members of series $I$ and $I$ have been studied and the structural characteristics have been measured. An hexagonal three-dimensional network in serie II exists for one homologous, the structure of which is not yet understood $/ 7 /$. In order to compare theese compounds we have define a parameter B which measure the core to chain volume ratio as the number of carbon atoms of the paraffinic moiety divided by the core length measured in rings units.

\section{III-STRUCTURAL CHARACTERISTICS OF THE CUBIC PHASE}

Powder patterns have been performed with a Guinier camera equipment and a linear focus. The patterns are all similar and consistent with our first observations. Therefore we assume that the space group is I $\mathrm{m} 3 \mathrm{~m}$ for all these phasmidic cubic phases. The lattice cell constants are reported on table I.

Table I. Lattice constants of the mesophases of pure compounds and binary mixtures. In this last case, since, there is no ambiguity, the chain length of two components has not been reported.

\begin{tabular}{|c|c|c|c|c|c|}
\hline \multirow{2}{*}{ Compound } & \multicolumn{2}{|c|}{ 3D lattices } & \multicolumn{2}{|c|}{ 2D lattices } & \multirow{2}{*}{$\begin{array}{c}\text { SmC layer } \\
\text { thickness }\end{array}$} \\
\hline & space group & $\begin{array}{c}\text { unit cell } \\
\mathbf{a}\end{array}$ & symmetry & $\begin{array}{c}\text { unit cell } \\
a ; b ;(n m) ; Y^{\circ n} \\
\end{array}$ & \\
\hline I 11 & $1 \mathrm{~m} 3 \mathrm{~m}$ & 16.20 & Hex II & 13.5 & \\
\hline I 12 & $"$ & 17.18 & Hex II & 12.93 & \\
\hline II 10 & $"$ & 18.26 & Oblique & $9.23 ; 5.26 ; 97.9$ & 4.02 \\
\hline III 8 & $"$ & 15.57 & & & \\
\hline IV 12 & $"$ & 16.93 & & & \\
\hline v 18 & la $3 d$ & 11.60 & & & \\
\hline $0.79 \quad I I I / 0.21 \mathrm{~V}$ & $1 \mathrm{~m} 3 \mathrm{~m}$ & 16.43 & & & \\
\hline $0.61111 / 0.39 v$ & $"$ & 16.57 & & & \\
\hline $0.30 \quad I I I / 0.70 \mathrm{~V}$ & $"$ & 16.93 & & & \\
\hline $0.10 \quad 111 / 0.90 \mathrm{~V}$ & $"$ & 17.13 & & & \\
\hline $0.665 \mathrm{~V} / 0.335 \mathrm{VI}$ & $"$ & 16.52 & & & \\
\hline $\mid \begin{array}{c}0.795 \mathrm{~V} / 0.205 \mathrm{~V} \\
+0.205 \text { VI } 11\end{array}$ & " & 15.96 & & & \\
\hline
\end{tabular}

The hex II phase of the undecyloxy derivative $I_{11}$ have been identified on single crystals examined in a point focus geometry,powder patterns, which needs few hours of exposure, can be obtained only for the same phase of $\mathbf{I} 12$. The previously proposed complex structure /13/ is consistent with these new 
results since the hexagonal lattice has a parameter of $130 \AA$.

In order to have a better understanding upon the cubic I $\mathrm{m} 3 \mathrm{~m}$ mesophase, we have studied some binary mixtures of the rod-like molecule p-4-(octadecyloxy-4-nitro-3, phenyl)-p' benzoic acid (V18)/14/ with the compound $\mathrm{III}_{8}$ and with a non mesomorphic benzoic acid substituted in 3 and 5 (VI11) by undecyloxy chain /15/. In the first case the I $\mathrm{m} 3 \mathrm{~m}$ mesophase accepts at least 80 weight $\%$ of rod-like compound, the lattice parameter increases with the rod-like compound amount. In the second binary mixture a I m $3 \mathrm{~m}$ mesophase is induced for an amount of 15- 35 weight\% of disubstituted benzoic acid in the rod-like one. The lattice parameters are still of the order of $15 \mathrm{~nm}$ in both cases. The structural features of all these mesophases are reported on table I.

\section{IV-DISCUSSION}

Among the periodic minimal surfaces dividing the space into two interwowen infinite labyrinthes, the Schwarz surface $P$ gives a suitable representation of the cubic phase of symmetry space group I $\mathrm{m} 3 \mathrm{~m}$ in lyotropic systems $/ 2 /$. A molecular layer of phasmids is equivalent to a lipidic bilayer; since the cross section of a molecule increases going from the median surface (localised in the aromatic medium) towards the external surfaces where the methyl end groups are localised such a molecular layer fill each labyrinth of the cubic network with its methyl end groups sitting on the interface built by the $\mathrm{P}$ surface. The cubic unit cell contains $\mathrm{N}_{0}$ molecules:

$$
\mathrm{N}_{0}=\rho \mathrm{a}^{3} / \mathrm{M}
$$

where $\rho$ is the density, $M$ the molecular mass and a the lattice parameter. The minimal surface $S_{o}$ per unit cell is also related to the lattice parameter a by a geometric factor $\sigma$ :

$$
S_{0}=\sigma a^{2}
$$$$
(\sigma=2.345 \text { for the P Shwartz surface } / 16 /)
$$

If each molecule has $m$ chains and if all their methyl end groups are localised on the $P$ surface, there are $\mathrm{N}_{0} / 2 \mathrm{n}$ chains on each side of $S_{0}$. Under this assumption, the mean area per chain $2 \sigma \mathrm{M} / \mathrm{npa}$ with $\rho=1 \mathrm{~g} \mathrm{~cm}^{-3}$, is of the order of $0.14 \mathrm{~nm}^{2}$ which is unrealistic. Moreover the mean distance between the two labyrinthes $a / 2$ is larger than the molecular length. Therefore the molecular organization is of complex kind /1/: each labyrinth is divided into two layers by an intermediate interface parallel to the $P$ surface lying at a distance $\alpha$ a of it. For small value of $\alpha$ the surface $S_{1}$ per unit cell of this intermediate interface is related to $S_{0} / 16 \%$

$$
S_{1}=S_{0}+2 \pi \chi \alpha^{2} a^{2}
$$

Where $\chi$ is a geometrical factor related to the genus of the minimal surface (in our case $\chi=-4$ ). The methyl end groups of the molecules of each labyrinth can be either on each side of $S_{1}$ or on one side of $S_{0}$. Therefore the mean area per chain $A$ is a function of $\alpha$ through the relation:

$$
m N_{0} A=6 \sigma a^{2}+8 \pi \chi \alpha^{2} a^{2}
$$

Replacing $\mathrm{N}_{0}$ by its value gives:

$$
\mathrm{A}=\left(6 \sigma+8 \pi \chi \alpha^{2}\right) \mathrm{M} / \mathrm{m \rho a}
$$

A better description of the siructure would imply the knowledge of the repartition of molecules into the two parts of the labyrinth and the repartition of the chains on the external and the intermediate surface of a labyrinth.In fact electronic density profiles can be deduced from diffracted intensities measurements using direct methods of structure resolution /3/. In a preliminary approach, using crude assumptions, we attempt to test if a model of complex labyrinthes separated by the minimal surface $P$ (Fig.2)will provide reasonable values of $A$ and $\alpha$ that can be compared to the same values calculated for the SmC and for the hexagonal columnar phases of phasmidic compounds. Firstly, we assume that the molecular density is the same on each side of $S_{1}$. This assumption is 
supported by our X-ray data. The first diffraction rings are non-measurable and the highest intensity peaks correspond to lattice spacing between. $a / \sqrt{ } 12$ and $a / \sqrt{18}$. Thus, the main contribution to the density modulation arised from intralayer terms and the electronic density is nearly equal on the two sides of the intermediate surface.

Secondly, we assume that the methyl end groups have the same area whatever is the interface. This second assumption has consequences at a microscopic level : With symmetrical molecules in a global trans conformation the mean area per chain must be higher on $S_{0}$ than on the external side of $S_{1}$; therefore our second assumption implies that the external sublayer of a labyrinth contains either oriented asymmetrical (three-chains) molecules or symmetrical molecules with their core in a bent conformation see fig. 2 . Let us remark that bent conformations were proposed in order to explain the structural features of the columnar hexagonal phase of phasmids $/ 17 /$.Moreover the cubic phase is found for biforked molecules with a rather flexible central core.

If $N_{1}$ is the number of molecules of the external part of the labyrinth the uniform density assumption links $\mathrm{N}_{1}$ and $\alpha$ :

$$
N_{1} M=1 / 2 \rho\left(S_{1}+S_{0}\right) \alpha a=\rho \alpha a^{3}\left(\sigma+\pi \chi \alpha^{2}\right)
$$

The uniform area assumption gives a second relation between $\mathrm{N}_{1}$ and $\alpha$ :

$$
\begin{aligned}
& \mathrm{A}=\left(\mathrm{S}_{1}+\mathrm{S}_{0}\right) / \mathrm{mN}_{1}=\left(4 \mathrm{~S}_{1}+2 \mathrm{~S}_{0}\right) / \mathrm{mN}_{0} \\
& \mathrm{~N}_{1}=\rho \mathrm{a}^{3}\left(\sigma+\pi \chi \alpha^{2}\right) / \mathrm{M}\left(3 \sigma+4 \pi \chi \alpha^{2}\right)
\end{aligned}
$$

Combining the volume (1) and area (2) conditions one obtains a third degree equation for $\alpha$ :

$$
4 \pi \chi \alpha^{3}+3 \sigma \alpha-1=0
$$

The two positive solutions are $\alpha=0.196$ and $\alpha=0.236$. The mean chain area $A$ is proportional to the inverse of $\alpha$ :

$$
\mathrm{A}=2 \mathrm{M} / \mathrm{mpa} \alpha
$$

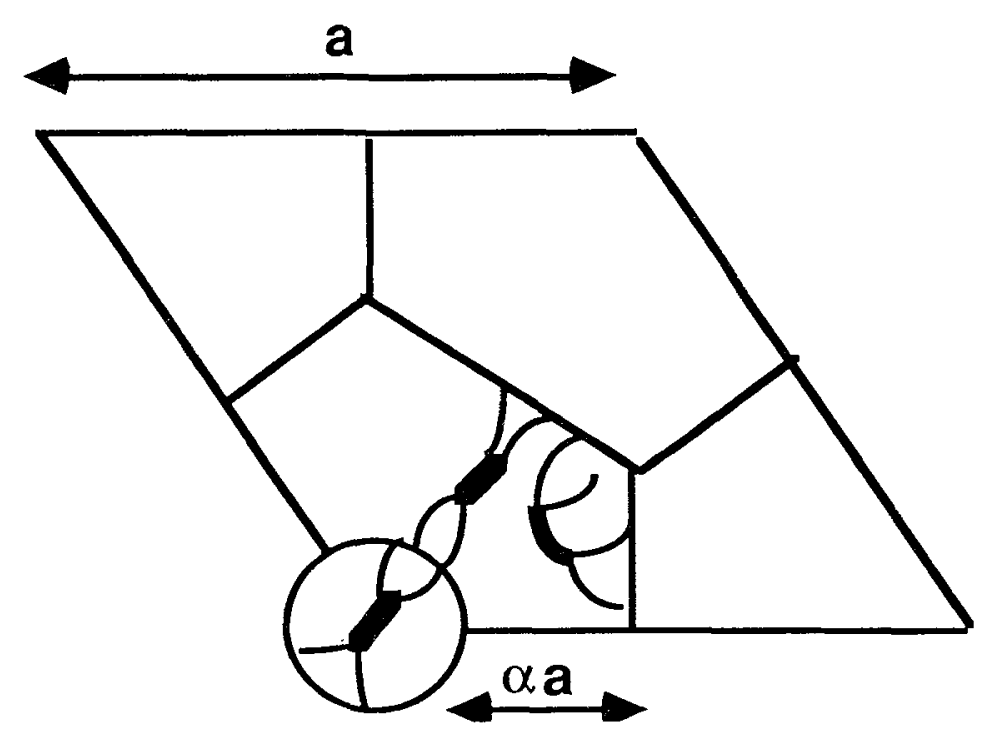

Fig. 2. Schematic representation of the molecular organization of the complex columnar hexagonal mesophases. Two distinct molecular layers, the inner one is a cylinder while the outer one is limited on one side by the boundary of the inner layer and on the other side by the prismatic minimal surface. The cubic phase shows the same topology. 
We have reported in table II the value of the mean area per chain A for each value of $\alpha$.For four chains phasmids we choose the smallest value of $\alpha$ since it corresponds to the smallest proportion of bent molecules. Therefore the external shell thickness is nearly equal to the. SmC layer thickness (Respectively 3.58 and $4.02 \mathrm{~nm}$ for $\mathrm{II}_{10}$ ). If the external sublayer has locally a SmC organization,a symmetry arguement implies that the bent molecules are located near the threefold axes. The amount of bend molecules in this sublayer is:

$$
\left(S_{0}-S_{1}\right) /\left(S_{1}+S_{0}\right)=(3 \sigma-1) /(\sigma+1) \text { i.e. } 0.26 \text { for } \alpha=0.196
$$

The proportion of molecules of the inner shell is :

$$
2\left(\mathrm{~N}_{0}-\mathrm{N}_{1}\right) / \mathrm{N}_{0}=(1-\sigma) / 2=0.27 \text { for } \alpha=0.196
$$

Table II. Mean chain areas in different mesophases. The number of chains of a binary mixture is a mean molecular value;inside brackets the average value takes into account pairs of acids (V or VI). The two solutions of our model for the $\mathrm{Im} 3 \mathrm{~m}$ stucture have been reported and the best.one is in bold character. Notice that a mean chain number of three per pair of acid or per phasmidic molecule

\begin{tabular}{|c|c|c|c|c|c|c|c|}
\hline \multicolumn{3}{|c|}{ System } & & \multicolumn{2}{|c|}{ Cubic I m $3 \mathrm{~m}$} & \multicolumn{2}{|c|}{ other mesophases } \\
\hline Composition & Chain & & $\begin{array}{c}\text { Mol Mass } \\
M\end{array}$ & $\begin{array}{l}A 1(\mathrm{~nm}) 2 \\
\alpha=.196\end{array}$ & $\begin{array}{l}\text { A2 }(\mathrm{nm}) 2 \\
\alpha=.236\end{array}$ & Phase & $A(n m) 2$ \\
\hline 111 & & 4 & 1208 & 0.317 & 0.263 & HexII & 0.288 \\
\hline $1 \quad 12$ & & 4 & 1264 & 0.312 & 0.259 & Hexll & 0.314 \\
\hline II 10 & & 4 & 1324 & 0.308 & 0.255 & Smc & 0.274 \\
\hline III 8 & & 4 & 1126 & 0.307 & 0.255 & & \\
\hline IV 12 & & 3 & 1093 & 0.366 & 0.303 & & \\
\hline v 18 & & 1 & 511 & & & la3d & 0.359 \\
\hline $0.79 \quad 111 / 0.21 \mathrm{~V}$ & 3.38 & $(3.76)$ & 999.3 & 0.305 & 0.253 & & \\
\hline $0.61 \quad I I 1 / 0.39 \mathrm{~V}$ & 2.83 & $(3.51)$ & 886.2 & 0.321 & 0.266 & & \\
\hline $0.30 \quad 111 / 0.70 \mathrm{~V}$ & 1.90 & $(2.92)$ & 696.1 & 0.367 & 0.305 & & \\
\hline $0.10 \quad 111 / 0.90 \mathrm{~V}$ & 1.30 & $(2.36)$ & 572.5 & 0.436 & 0.362 & & \\
\hline $0.665 \quad V / 0.335 \mathrm{VI}$ & 1.34 & $(2.68)$ & 494.6 & 0.379 & 0.315 & & \\
\hline $0.795 \mathrm{~V} / 0.205 \mathrm{VI}$ & 1.21 & $(2.42)$ & 501.0 & 0.440 & 0.366 & & \\
\hline
\end{tabular}
corresponds to the transition between these two solutions.

We have already discussed the structural features of the Hex I phase /11/ and our conclusion is that the section of a column is a cluster of four or five molecules with a five phenyl rings core (the cluster is larger for longer cores). Each cluster contains parallel rigid molecules the symmetry of the column is due to the orientational disorder between similar clusters in a column; another configuration have been proposed by Guillon et al. /17/ in which the external molecules of a cluster are bent. Anyway there are less than half of the total number of molecules in a bent conformation. If we assume 
that the internal shell of a labyrinth of the cubic structure has a similar organisation the total fraction of bent molecules does not exceed $36 \%$, which is less than in the simple hexagonal phase.

For a three-chains phasmid, we must take into account the disymmetry of the molecule. With a ratio $S_{0} / S_{1}=2$ the external sublayer will be totally polarized. If $\alpha=0.236$, the ratio $S_{0} / S_{1}=2.5$ but the amount of bent molecules is small. The proportion of molecules of the inner shell is $22 \%$ (such molecules are orientationally disordered,up and down, with respect to an axis perpendicular to $S_{1}$ ); $11 \%$ of the molecules have a bent conformation in the external shell and $68 \%$ of the molecules are in trans conformation with their one chain end pointing towards the intermediate surface. For $\alpha=0.196$ $S_{0} / S_{1}=1.7$ and there are no more bent molecules. However with $\alpha=0.236$ the mean area per chain is similar to that of the four chain derivatives and thus corresponds likely to the best model.

In binary mixtures, in absence of microsegregation, we can apply the same model provided we take a mean value for $M$ and $n$, (we consider that the acid compounds form dimeric units which can be disymetric in the mixtures of two different acids). The mean number of chains evolves between 4 and 2 per dimer. Without a microsegregation of the two species the results are comparable to that of pure phasmidic molecules. The segregation cannot be important as far as the proportion of molecules of the inner shell remains small. Let us remark that, in the mixtures, the $I \mathrm{~m} 3 \mathrm{~m}$ and $\mathrm{I}$ a $3 \mathrm{~d}$ phases coexist for a mean number of chain per dimer of about 2.3.

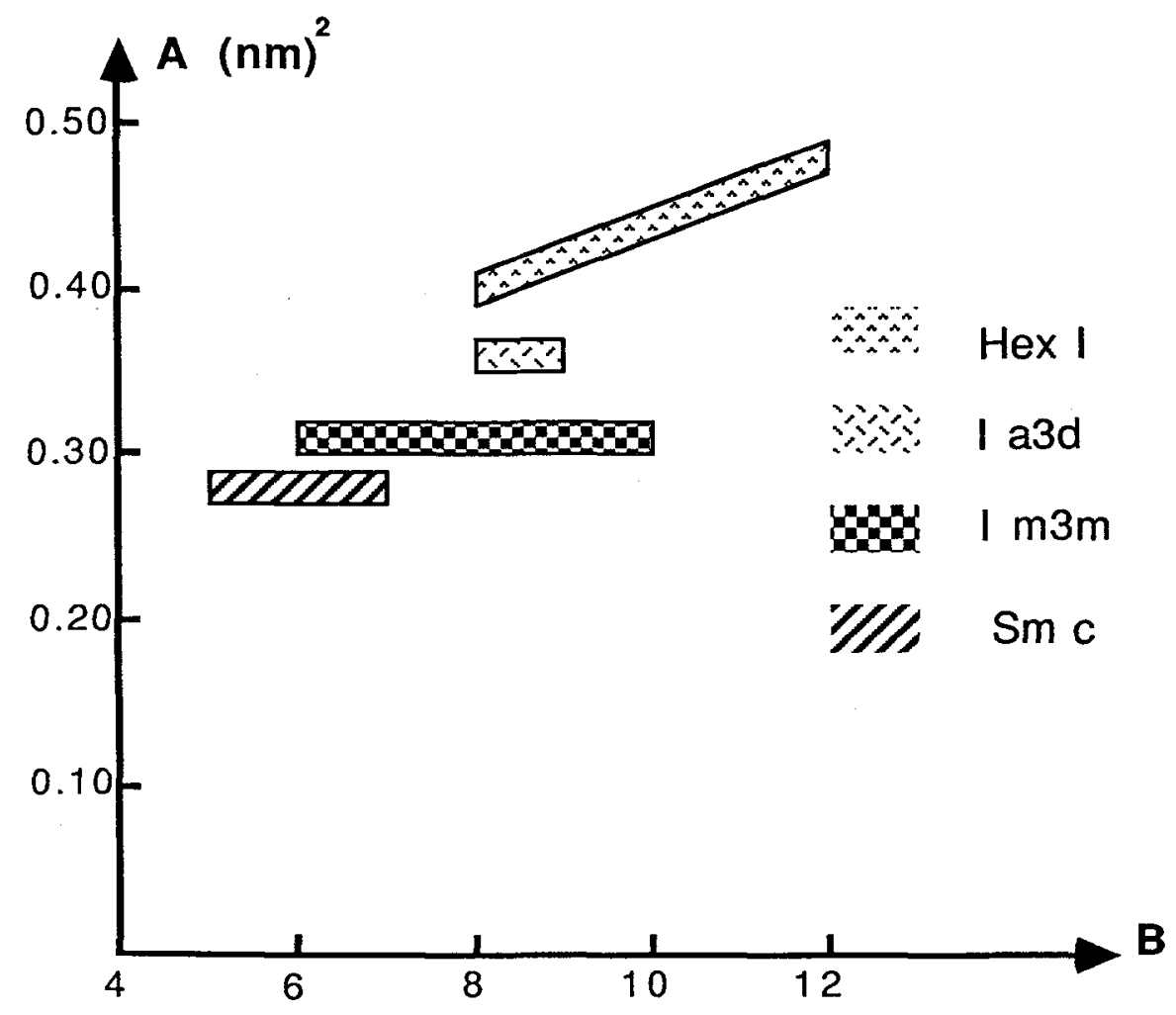

Fig. 3. Mean chain area in the different mesophases. $B=n m / N b$ of rings of the core.

A similar model can give a description of the molecular organisation of the Hex II mesophase of compound of series I. One obtains a unique solution with a mean area per chain which is nearly the same in the cubic and in the Hex II phase In the simpler structures (Cubic Ia3d, SmC and Hex I) the mean area per chain at the level of the methyl end groups (assuming a density of $1 \mathrm{~g} \mathrm{~cm}^{-3}$ for all the mesophases) is straightfully derived from the unit cell length. The Figure 3 gives the dependence of this area versus the above defined parameter B for the different phasmidic mesophases. This area 
increases from the lamellar to the cubic and then to the hexagonal mesophase,and from the complex Im3m phase to the simple Ia3d one.

\section{V-CONCLUSION}

We have shown that periodic minimal balance surfaces can provide a good description of the cubic mesophase of phasmids. The structural parameters derived from a double layer model are consistent with the theoretical approach given for lyotropic systems[18]. However the complex double layer organisation of this cubic mesophase underline the role of topological defects such as disclination lines upon the polymorphism. The Hex II mesophase can be described as well as periodic arrays of focal conics while the I $\mathrm{m} 3 \mathrm{~m}$ phase corresponds to a more complex regular network of both disclination lines and point singularities. Moreover these two phases, which occur simultaneously, corresponds to the same value of the chain area. The spontaneous transformation of the second into the first one might be driven by the interaction between neighbouring disclinations. The sequence of hexagonal, rhomboedral, and cubic phases find in similar compounds may be another illustration of this idea.

\section{ACKNOWLEDGEMENTS}

We thank Eric Bolzonello and Bernard Hehlen for their active participation to the X-ray diffraction experiments.

\section{REFERENCES}

/1/ Luzzati, V., In Biological Membranes edited by D. Chapman Ac Press N. York (1968).

12/ Sadoc, J.F., Charvolin, J., J. de Physique 47 (1986) 683.

CharvolinJ., Sadoc, J.F., J. de Physique 48 (1987) 1559.

13/ Mariani, P., Luzzati, V., Delacroix, H., J. Mol. Biol. 204(1988) 165.

14/ Billard, J., C.R. Acad. Sci. Paris, Série II, 305 (1987) 843.

15/ Malthète, J., Levelut, A.M., N'Guyen Huu Tinh, J. de Physique Lett. 46 (1985) L 875.

16/ Hendrikx, Y., Levelut, A.M., Mol. Cryst. Liq. Cryst. 165 (1988) 223.

I7I Fang, Y., Levelut, A.M., Destrade, C., Liq. Crystals, I (1990) 265.

18/ N'Guyen Huu Tinh, Destrade, C., Levelut, A.M., Malthète, J., J. de Physique 47 (1986) 543.

19/ Destrade, C., N'Guyen Huu Tinh, Alstermark, C., Lindsten, G., Nilsson, M., Otterholm, B., Mol. Cryst. Liq. Cryst. 180 B (1990) 265.

/10/ Alstermark, Eriksson, N., Nilsson,M, Destrade,C, N'Guyen Huu Tinh, Liq. Cryst. 8 (1990) 75.

111/ Malthète, J., N'Guyen Huu Tinh, Private communication.

/12/ Levelut, A.M., Fang, Y., Destrade, C., Liq. Crystals 4 (1989) 561.

/13/ Levelut, A.M., Malthète, J., Destrade, C., N'Guyen Huu Tinh, Liq. Crystals 2 (1987) 877.

/14/ Demus, D., Marzotko, D., Sharma, N.K., Wiegeleben, A., Kristall und Technik 15 (1980) 331.

115/ The compound $\mathrm{V}_{18}$ comes from $\mathrm{C}$. Germain and the compound $\mathrm{VI}_{11}$ from J. Malthète; we acknowledge both of them for providing us these samples.

/16/ Barois, P., Hyde, S., Ninham, B., Dowling, T., Langmuir 6 (1990) 1136

/17/ Guillon, D., Skoulios, A., Malthète, J., Europhys. Lett. 3 (1987) 67. 